\title{
Fourier series And Fourier Transform
}

\author{
Mr. Karan Asher \\ Electronics And Telecommunication, K.J.Somaiya College Of Engineering University Of Mumbai, India
}

\begin{abstract}
An introduction to Fourier Series and Fourier Transform is the topic of this paper. It deals with what a Fourier Series means and what it represents. The general form of a Fourier Series with a provision for specific substitution has also been mentioned.

The paper also includes a brief overview of Fourier Transform. The use of Fourier Transform to convert a time domain function into a frequency domain equivalent has also been shown. A method of converting the continuous Fourier Transform into a discrete form and thus obtaining the Discrete Fourier Transform has also been discussed. A few practical life application of Fourier analysis have been stated.
\end{abstract}

\section{Introduction}

This paper deals with Fourier Series on representing a periodic function in the form of a sum of sinusoids and Fourier Transform which helps convert a time domain function into a frequency domain function. A series is the addition of the terms of a sequence. The series,

$$
\frac{a_{0}}{2}+\left(a_{1} \cos x+b_{1} \sin x\right)+\left(a_{2} \cos 2 x+b_{2} \sin 2 x\right)+\cdots\left(a_{n} \cos n x+b_{n} \sin n x\right)
$$

This can be in general written as,

$$
f(x)=\frac{a_{0}}{2}+\sum_{n=1}^{\infty}\left(a_{n} \cos n x+b_{n} \sin n x\right)
$$

Where a's and b's are all constants can be represented as a specific trigonometric series. The function $\mathrm{f}(\mathrm{x})$ represented by the above equation is a periodic function of period $2 \pi$. The expansion of $f(x)$ in the form of the above series is classified as Fourier Series. Any periodic function of period $2 \pi$ satisfies a specific set of conditions known as the Dirichlet's conditions.

\subsection{Dirichlet's Coditions}

\section{Fourier Series Specifics}

Consider $\mathrm{f}(\mathrm{x})$ to be any random function. It can be expressed as Fourier Series if,

1) $f(x)$ and its integrals are finite and single valued

2) $f(x)$ has discontinuous finite number

3) $f(x)$ has finite number of maxima and minima.

If a function is discontinuous, it can still be expressed as Fourier Series.

For example, if $\mathrm{x}=\mathrm{c}$ is the point of finite discontinuity, at this point, the series gives the arithmetic mean of the two limiting values as,

$$
f(c)=\frac{1}{2}\left[\lim _{x \rightarrow c^{-}} f(x)+\lim _{x \rightarrow c^{+}} f(x)\right]
$$

\subsection{Evaluation Of Coefficients}

The coefficients $a_{0}, a_{n}, b_{n}$ of the Fourier Series can be found using the following formulae,

$$
\begin{gathered}
a_{0}=\frac{1}{\pi} \int_{p}^{p+2 \pi} f(x) d x \\
a_{n}=\frac{1}{\pi} \int_{p}^{p+2 \pi} f(x) \cos n x d x \\
b_{n}=\frac{1}{\pi} \int_{p}^{p+2 \pi} f(x) \sin n x d x
\end{gathered}
$$

Thus, a Fourier Series represents the sum of a series of trigonometric expressions used in the analysis of periodic functions.

\subsection{Generalised Form}

The above mentioned formulae and series are for those specific functions which are periodic in the interval $(0,2 \pi)$. A more generalised form of Fourier Series can be expressed as, 


$$
f(x)=\frac{a_{0}}{2}+\sum_{n=1}^{\infty}\left(a_{n} \cos \frac{n \pi x}{L}+b_{n} \sin \frac{n \pi x}{L}\right)
$$

Thus, the above mentioned case is one with $\mathrm{L}=2 \pi$. We can define a Fourier Series for functions in the intervals like $(0,21),(0,2 \pi),(-l, l),(-\pi, \pi)$.

\subsection{Parseval's Identity}

If a function $\mathrm{f}(\mathrm{x})$ converges uniformly in $(\mathrm{c}, \mathrm{c}+2 \mathrm{l})$, then a special identity known as the Parseval's identity is applicable. The same can be represented as,

$$
\frac{1}{2 l} \int_{c}^{c+2 l}[f(x)]^{2} d x=\frac{a_{0}^{2}}{2}+\frac{1}{2} \sum_{n=1}^{\infty}\left(a_{n}^{2}+b_{n}^{2}\right)
$$

This is known as the Parseval's Identity for the function $\mathrm{f}(\mathrm{x})$ in the interval $(\mathrm{c}, \mathrm{c}+2 \mathrm{l})$.

\section{Half Range Series}

A complete Fourier Series is one which contains both the sine as well as the cosine terms. On the other hand, a Half Range Series would be one which eliminates either the sine or the cosine term of the series due to certain properties of the function itself.

The generalised form of the Fourier Series in the interval $(-l, l)$ is expressed as,

With the value of the coefficients evaluated as,

$$
f(x)=\frac{a_{0}}{2}+\sum_{n=1}^{\infty}\left(a_{n} \cos \frac{n \pi x}{L}+b_{n} \sin \frac{n \pi x}{L}\right)
$$

$$
\begin{gathered}
a_{0}=\frac{1}{l} \int_{-l}^{l} f(x) d x \\
a_{n}=\frac{1}{l} \int_{-l}^{l} f(x) \cos \frac{n \pi x}{l} d x \\
b_{n}=\frac{1}{l} \int_{-l}^{l} f(x) \sin \frac{n \pi x}{l} d x
\end{gathered}
$$

Now, if the function $\mathrm{f}(\mathrm{x})$ is even, then the coefficient $b_{n}$ will turn out to be zero since it will be an integral of a product of odd and even function which will eventually be an odd function within the limits $(-l, l)$. Thus the series would then be represented as,

$$
f(x)=\frac{a_{0}}{2}+\sum_{n=1}^{\infty}\left(a_{n} \cos \frac{n \pi x}{L}\right)
$$

Thus, the series now does not have any sine term in the expression and this is called the Half Range Cosine Series since it contains only the cosine terms and eliminates all the sine terms.

Similarly, if the function $\mathrm{f}(\mathrm{x})$ is an odd function, then the coefficients $a_{0}$ and $a_{n}$ turn out to be zero since $a_{0}$ represents an odd function within the limits $(-l, l)$ and $a_{n}$ represents a product of odd and even function which will eventually be an odd function within the limits $(-l, l)$. Thus, the series would then be represented as,

$$
f(x)=\sum_{n=1}^{\infty}\left(b_{n} \sin \frac{n \pi x}{L}\right)
$$

Thus, the series now does not have any cosine term in the expression and this is called the Half Range Sine Series since it contains only the sine terms and eliminates all the cosine terms.

\section{Fourier Transform}

The Fourier Transform is a type of mathematical transform. The Fourier Transform transforms a nonperiodic function $\mathrm{f}(\mathrm{t})$ in time domain into a function $\mathrm{F}(\mathrm{s})$ in its corresponding frequency domain. It decomposes any given into a sum of sinusoids. The Fourier series is a method of expressing a periodic function as a sum of sinusoids. The Fourier Transform is thus an extension of this idea to non-periodic functions as well. This makes the Fourier transform immensely effective in conduction of heat, wave propagation, digital signal processing, image processing, filtering, etc.

\subsection{Definition And Meaning Of Fourier Transform}

If a function $f(x)$ is defined on $(-\infty, \infty)$, is piecewise continuous in each finite interval and is absolutely integrable in $(-\infty, \infty)$, then the Fourier Transform of $f(x)$ can be expressed as, 


$$
F(s)=\frac{1}{\sqrt{2 \pi}} \int_{-\infty}^{\infty} f(x) e^{i s x} d x
$$

The above calculated function $F(s)$ consists of the frequency components of the time domain function $f(x)$. Thus, if you take the transform of a sine wave, a single spike will be observed at the frequency (positive and negative) of the sine wave. This in turn means that all the energy of the sine wave is concentrated at that particular frequency. Negative frequencies have no physical meaning in real life.

If the time domain function $\mathrm{f}(\mathrm{x})$ is a real function, then, its corresponding Fourier Transform $\mathrm{F}(\mathrm{s})$ will be an even function. Thus, the positive frequencies will always mirror the negative frequencies. Therefore, a time domain function $\mathrm{f}(\mathrm{x})$ and its corresponding frequency domain function are duals of each other.

The above can be diagrammatically represented as shown. Consider a simple square pulse of amplitude A.

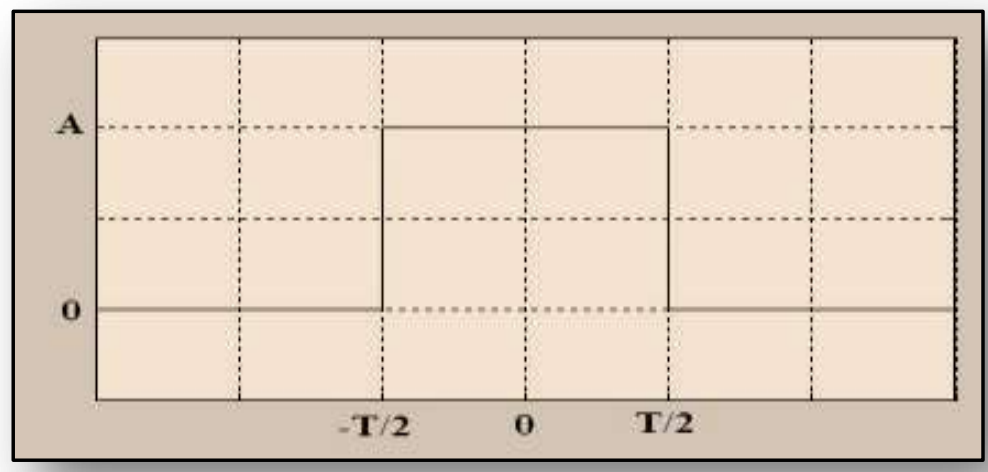

Fig. 1

This square pulse represents a time domain function $\mathrm{f}(\mathrm{t})$ with amplitude A. with the application of equation (7) on this time domain function, its corresponding frequency domain equivalent is represented diagrammatically as,

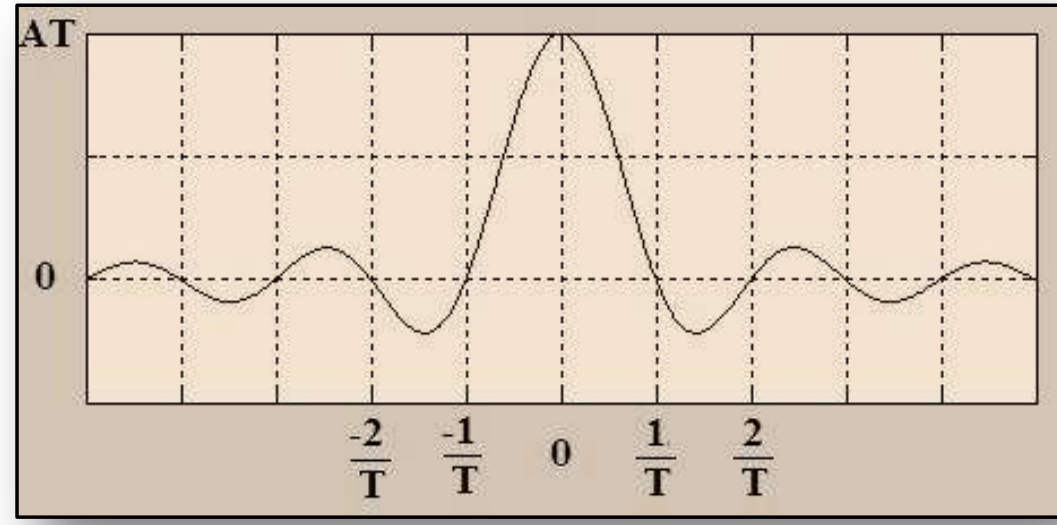

Fig. 2

\subsection{Inverse Fourier Transform}

The Inverse Fourier Transform is a method of converting a frequency domain function back to its time domain form. If $F(s)$ is the Fourier Transform of $f(x)$ and if $f(x)$ satisfies Dirichlet's conditions in every finite interval $(-l, l)$ and if $\int_{-\infty}^{\infty}|f(x)| d x$ is convergent, then

Represents the inverse Fourier Transform of F(s).

$$
f(x)=\frac{1}{\sqrt{2 \pi}} \int_{-\infty}^{\infty} F(s) e^{-i s x} d x
$$

\section{Discrete Fourier Transform}

We have already seen now that the Fourier Transform helps convert a time domain function into its corresponding frequency domain equivalent. But however, $\mathrm{F}(\mathrm{s})$ is a continuous function of frequency and is therefore not convenient form of data to be handled by a digital system. Hence, a function $\mathrm{f}(\mathrm{t})$ is represented by 
the samples of its spectrum $\mathrm{F}(\mathrm{s})$. Such a frequency domain representation leads to the Discrete Fourier Transform (DFT), which is more convenient to handle for the frequency analysis of discrete time signals.

\subsection{Dft Definition}

A finite duration sequence of length $\mathrm{L}$ has a Fourier Transform which is given by,

$$
F(s)=\sum_{n=0}^{L-1} f(n) e^{i \omega t}, \quad 0 \leq \omega \leq 2 \pi
$$

Where the upper and lower indices in the summation reflect the fact that $\mathrm{x}(\mathrm{n})=0$ outside the range $0 \leq n \leq L-1$. When we sample $\mathrm{F}(\mathrm{s})$ at equally spaced frequencies $2 \pi k / N, \mathrm{k}=0,1 \ldots \mathrm{N}-1$, where $N \geq L$,

$$
F(k)=\sum_{n=0}^{N-1} f(n) e^{-\frac{i 2 \pi n}{N}}, \quad k=0,1, \ldots, N-1
$$

Where for convenience, the upper index in the summation has been increased to $\mathrm{N}=1$ since $\mathrm{x}(\mathrm{n})=0$ for $n \geq L$. The equation (10) helps convert a finite sequence into a set of frequency samples of length N. Since the frequency samples are obtained by evaluating the Fourier Transform into a set of equally spaced frequencies, equation (10) is thus called the Discrete Fourier Transform of $f(n)$. The expression that helps us recover the sequence $f(n)$ from the frequency samples is given by,

$$
f(n)=\frac{1}{N} \sum_{k=0}^{N-1} F(s) e^{\frac{i 2 \pi n}{N}}
$$

And is thus called the Inverse Discrete Fourier Transform (IDFT)

\section{Applications}

Fourier analysis is put to very effective use in practical applications in almost every field of science and engineering. A few examples on where is Fourier transform is used have been mentioned. Fourier analysis finds applications in Signal processing, Digital processing, Image processing, Digital filtering, Conduction of heat, Wave propagation. It would not be wrong to say that Fourier analysis does contribute to nothing but almost all branches of Applied Sciences.

\section{Conclusion}

This paper thus, consisted of a brief overview of what Fourier analysis is and what does it mean. A complete description of Fourier Series was discussed along with the evaluation of its coefficients and certain properties and identities. Also, it included the description of the use of Fourier Transform and its immense potential in converting a time domain function into its frequency domain equivalent. Expanding the idea of the Fourier Transform, Discrete Fourier Transform was also discussed with its advantage of having converted the Fourier Transform which is continuous into a set of frequencies which are discrete. It goes without saying that every branch of Applied Sciences does use Fourier analysis.

\section{Acknowledgement}

I hereby take the opportunity to thank everyone who helped me make this paper. My college professors PROF. ALGONDA DESAI, PROF. YOGESH THAKKAR, a few of my classmates and my brother, all had helped me make this paper and a great vote of thanks to all of them.

[1] www.tutorial.math.lamar.edu

\section{References}

[2] www.sunlightd.com/fourier/3

\section{Reference Authors}

1. Prof. Algonda Desai, Msc.Mathematics

2. B.V.Ramamna, Higher Engineering Mathematics, Tata Mc-Graw Hill Publication

3. Dr.B.S.Grewal, Higher Engineering Mathematics, Khanna Publication

4. Prof. P.N.Wartilar, Engineering Mathematics

5. Dr.Srivastava, Engineering Mathematics Ii 